\title{
3D FLAIR Cochlear Abnormality Does Not Predict on Sudden Sensorineural Hearing Loss Prognosis: A Retrospective Cohort Study
}

\author{
Geun Cheol Shin ${ }^{1}$ (D) Ara Cho ${ }^{2}$ (D) Min Cheol Jeong ${ }^{2}$, Beom Seok Sohn ${ }^{3}$, and Seong Hoon Bae ${ }^{2}$ (D) \\ ${ }^{I}$ Department of Otorhinolaryngology, Gangnam Severance Hospital, Yonsei University College of Medicine, Seoul; and \\ Departments of ${ }^{2}$ Otorhinolaryngology and ${ }^{3}$ Radiology and Research Institute of Radiological Science, \\ Yonsei University College of Medicine, Seoul, Korea \\ $3 \mathrm{D}$ 플레어 이미지에서의 달팽이관 이상소견과 돌발성 난청의 예후의 상관관계: 후향적 코호트 연구 \\ 신근철 $^{1} \cdot$ 조아라 $^{2} \cdot$ 정민철 $^{2} \cdot$ 손범석 $^{3} \cdot$ 배성훈 $^{2}$ \\ ${ }^{1}$ 연세대학교 의과대학 강남세브란스병원 이비인후과, 연세대학교 의과대학 ${ }^{2}$ 이비인후과학교실, ${ }^{3}$ 영상의학교실
}

\author{
Received May 17, 2021 \\ Revised June 18, 2021 \\ Accepted July 27, 2021 \\ Address for correspondence \\ Seong Hoon Bae, MD \\ Department of Otorhinolaryngology, \\ Yonsei University \\ College of Medicine, \\ 50-1 Yonsei-ro, Seodaemun-gu, \\ Seoul 03722, Korea \\ Tel $+82-2228-3611$ \\ Fax +82-2-393-0580 \\ E-mail bshsap1@yuhs.ac
}

Background and Objectives Previous studies reported abnormalities in MRI as a poor prognostic indicator of sudden sensorineural hearing loss (SSNHL). Since abnormalities in three-dimensional (3D) fluid-attenuated inversion recovery (FLAIR) are strongly correlated with the initial hearing function, the prognostic value of the 3D FLAIR images should be carefully evaluated to avoid collinearity. We aimed to evaluate abnormalities on the 3D FLAIR images as an independent prognostic factor in the matched SSNHL groups.

Subjects and Method We retrospectively reviewed medical records of 179 patients with SSNHL who underwent temporal MRI, including the 3D FLAIR sequence, between January 2015 and December 2019. Patients were divided based on the presence of cochlear abnormalities on the 3D FLAIR images. Hearing prognosis was evaluated with and without matching for initial hearing and treatment interval.

Results The groups were similar in sex $(p=0.091)$, age $(p=0.925)$, treatment interval $(p=$ $0.216)$, and MRI interval $(p=0.828)$. Notably, patients with cochlear abnormalities on the 3D FLAIR images showed distinctly more severe hearing loss $(p<0.001)$ at the initial pure tone average (PTA) assessment and poorer outcomes $(p<0.001)$ compared to those without abnormality. After matching for initial hearing and treatment interval, the hearing outcome, measured by PTA, was similar between the groups ( $p=0.681)$.

Conclusion Cochlear signal abnormality in 3D FLAIR MRI was associated with poor initial hearing. However, it did not affect hearing recovery outcomes when the groups were matched.

Korean J Otorhinolaryngol-Head Neck Surg 2022;65(3):143-9

\section{Introduction}

Sudden sensorineural hearing loss (SSNHL) is defined as

This is an Open Access article distributed under the terms of the Creative Commons Attribution Non-Commercial License (https://creativecommons.org/licenses/by-nc/4.0) which permits unrestricted non-commercial use, distribution, and reproduction in any medium, provided the original work is properly cited. an acute sensorineural hearing loss of at least $30 \mathrm{~dB}$ over three contiguous frequencies occurring within 3 days. The annual incidence of SSNHL is reported to be 5-20 cases per 100000 people. ${ }^{1)}$ The causes of SSNHL are still not entirely clear, with as many as $90 \%$ of cases considered idiopathic. ${ }^{2)}$ The standard treatment for SSNHL has been oral administration or intra- 
tympanic injection of corticosteroids, ${ }^{1,3,4)}$ with both showing similar results. ${ }^{3,5)}$ Many studies reported on the prognostic factors of SSNHL, including neutrophil/lymphocyte ratio, initial hearing functions, early treatment response, abnormalities in MRI, and others. ${ }^{6}$ )

Abnormalities in MRI were reported in 27\%-53\% of SSNHL cases. $^{7)}$ Fluid-attenuated inversion recovery (FLAIR) sequence MRI was used in many studies, and it was reported that signal enhancement in the cochlea was associated with poor prognosis and poor initial hearing function. ${ }^{3,89}$ Signal enhancement in the cochlea of the affected side in three-dimensional (3D) FLAIR is known to represent methemoglobin or proteinaceous exudate in the inner ear fluid space. ${ }^{7)}$ The poor prognosis and poor initial hearing function can be partially explained by a severe disruption of the blood-labyrinthine barrier (BLB) that can cause massive infiltration of methemoglobin or proteinaceous exudate. Poor initial hearing function in 3D FLAIRpositive SSNHL patients has been consistently reported. However, the prognosis of these patients seems to vary from study to study. For instance, Berrettini, et al. ${ }^{8)}$ and Lee, et al. ${ }^{10)}$ failed to find a significant relationship between 3D FLAIR abnormalities and hearing improvement.

The initial hearing function is generally regarded as an important prognostic factor for hearing recovery. ${ }^{11,12)}$ As the signal abnormality in 3D FLAIR was strongly correlated with the initial hearing function in previous studies, the prognostic utility of 3D FLAIR images should be carefully evaluated to avoid collinearity. Furthermore, the natural course of spontaneous recovery from SSNHL was reported to occur mainly within 2 weeks after symptom development. ${ }^{13)}$ The time interval from SSNHL onset to corticosteroid treatment was also reported to be an important prognostic factor, but some controversy remains. ${ }^{2)}$

Therefore, the present study aimed to investigate the prognostic value of 3D FLAIR images independently of possible confounding factors. Initial hearing and treatment intervalmatched patients were analyzed to minimize the confounding factors. We believe this study would help elucidate the prognostic utility of 3D FLAIR for SSNHL patients.

\section{Subjects and Methods}

\section{Patient enrollment}

This cohort study was approved by the Institutional Review Board (IRB) of the authors' affiliated hospital (4-2020-1090), and it was conducted in accordance with the principles out- lined in the Declaration of Helsinki. Using our institution's electronic database searching software, we screened 4643 patients who were diagnosed with SSNHL in our third referral university hospital between January 2015 and December 2019. Among them, 546 patients underwent temporal MRI including 3D FLAIR sequence. The medical charts of 546 patients were reviewed, and the patients who satisfied the inclusion criteria but not the exclusion criteria were enrolled for analysis. The inclusion criteria were: 1) aged 20-79 years (512 included); 2) pure tone average (PTA) in the unaffected side $<30 \mathrm{~dB}$ (467 included); 3) time interval to treatment from onset $<10$ days (321 included); 4) time interval to MRI from onset $<30$ days (284 included). The exclusion criteria were: 1) delayed treatment after the hospital visiting day (0 excluded); 2) recurring SSNHL (57 excluded); 3) abnormal findings on MRI that could affect hearing function (e.g., vestibular schwannoma, chronic otitis media) (40 excluded); 4) conductive hearing loss (e.g., abnormal drum finding, air-bone gap $>10 \mathrm{~dB}$ in PTA) (8 excluded). After the screening process, 179 SSNHL patients were enrolled in this study.

\section{SSNHL diagnosis, evaluation, and treatment}

Patients with sensorineural type hearing loss of at least 30 $\mathrm{dB}$ over three contiguous frequencies that occurred within 3 days were diagnosed as SSNHL. All patients were routinely investigated for ototoxic medication and noise exposure history. Otoscopic examination and pure tone audiograms were also routinely conducted and described in the medical chart. Temporal MRI, including 3D FLAIR images, was routinely recommended but selectively conducted on consenting patients. SSNHL patients were prescribed high dose oral corticosteroid therapy (methylprednisolone $48 \mathrm{mg}$ daily for 5 days, tapered out for the following 5 days) from the day of hospital visit. Few patients, likely to suffer from adverse side effects of systemic corticosteroid administration, were injected dexamethasone to the middle ear through the tympanic membrane. Three months after SSNHL onset, pure tone audiogram was conducted to evaluate the final hearing outcome. The degree of recovery was also evaluated at that time according to the Siegel's ${ }^{14)}$ criteria.

\section{Hearing test and MRI protocol}

Pure tone audiogram test was conducted in a sound-proof chamber. Bone-conduction and air-conduction hearing were evaluated. A threshold of over $120 \mathrm{~dB}$ in the air-conduction hearing test was regarded as scaled-out. PTA was defined as 
the average air-conduction thresholds at frequencies of 500, 1000, 2000, and $4000 \mathrm{~Hz}$. We used $120 \mathrm{~dB}$ for the scaled-out data when calculating the PTA.

MRI examinations were performed on 3-T scanners (Achieva [Philips Healthcare, Best, The Nethelands] and Ingenia [Philips Healthcare]). The following parameters were used for the 3D FLAIR: repetition time, 4800-8000 ms; echo time, 160-344 ms; flip angle, $90^{\circ}$; field of view, $180 \times 180 \mathrm{~mm}$; section thickness, $1.0-1.2 \mathrm{~mm}$; matrix, $256 \times 256$. The radiologist doctor interpreted pre-contrast 3D FLAIR images and gadolinium enhanced images. When the report indicated abnormal FLAIR findings, experienced ENT doctor performed a secondary inspection of the MRI examination.

\section{Statistical analysis}

Categorical variables were compared by Fisher's exact test. Student's t-test was used to compare continuous parameters between groups. To create two matched groups of "FLAIRpositive" and "FLAIR-negative," pairwise 1:1 matching was performed based on two variables, initial PTA (maximal difference, $5 \mathrm{~dB}$ ) and treatment interval (maximal difference, 3 days) in the patients with severe $(70-90 \mathrm{~dB})$ to profound (higher than $90 \mathrm{~dB}$ ) initial hearing loss. The two groups, each consisting of 23 matched patients, were created and compared using Student's t-test. One patient in the FLAIR-positive group was excluded from the matched group, as he/she failed to be paired based on initial PTA and treatment interval. Multivariate logistic regression was performed in 179 enrolled patients and 56 patients with profound initial hearing level. A $p$-value $<0.05$ was considered statistically significant. Statistical analyses were conducted using IBM SPSS Statistics for Windows, Version 25.0 (IBM Corp., Armonk, NY, USA).

\section{Results}

\section{Patient data categorized by cochlear abnormalities on 3D FLAIR images}

Of the 179 patients, 153 showed symmetric signal in the cochlea (FLAIR-negative group) and 26 showed signal increased cochlea in the affected side (FLAIR-positive group) on 3D FLAIR images (Table 1). There was no difference in sex $(p=0.091)$, age $(p=0.925)$, treatment interval $(p=0.216)$, and MRI interval ( $p=0.828)$ between the two groups. Notably, the group with signal increased cochlea in the affected side on 3D FLAIR images showed distinctly more severe hearing loss at the initial PTA $(p<0.001)$ and poorer outcomes $(p<$ $0.001)$ compared to the symmetric signal group. This result implies that an increased signal on 3D FLAIR images is strongly associated with the severity of initial hearing loss. Furthermore, we found significant correlation between initial PTA and cochlea signal increase in 3D FLAIR using Pearson correlation test (coefficient $r=0.378, p<0.001$ ). Consequently, the initial hearing should be carefully controlled to reveal the prognostic value of 3D FLAIR images.

\section{Hearing recovery in the matched groups}

As mentioned above, the initial hearing and treatment interval could be significant confounding factors affecting the final hearing outcome. To investigate the hearing outcome of SSNHL patients independently of the initial hearing and treatment interval, we matched the patients with severe to profound initial hearing for these factors (Table 2 and Fig. 1). After matching, the groups were similar in sex $(p=0.071)$, age ( $p=0.438)$, treatment interval $(p=0.666)$, MRI interval $(p=$ 0.133 ), and initial hearing $(p=0.617)$. The pure tone threshold at each of the tested frequencies (500, 1000, 2000, and 4000 $\mathrm{Hz}$ ) was similar between the groups (Fig. 2). Notably, the final hearing outcome in both groups was also similar in the

Table 1. Data of enrolled patients

\begin{tabular}{|c|c|c|c|}
\hline & FLAIR-negative $(n=153)$ & FLAIR-positive $(n=26)$ & $p$-value \\
\hline$\overline{\text { Sex (male:female) }}$ & $71: 82$ & $17: 9$ & 0.091 \\
\hline Age (year) & $49.6 \pm 12.7$ & $49.9 \pm 11.0$ & 0.925 \\
\hline Treatment interval (day) & $3.5 \pm 2.7$ & $4.2 \pm 3.1$ & 0.216 \\
\hline Intratympanic injection & $9(5.9)$ & $3(11.5)$ & 0.386 \\
\hline MRI interval (day) & $10.2 \pm 7.7$ & $10.6 \pm 7.9$ & 0.828 \\
\hline Initial PTA (dB) & $69.7 \pm 27.1$ & $100.1 \pm 22.6$ & $<0.001$ \\
\hline Final PTA (dB) & $38.4 \pm 27.4$ & $71.2 \pm 28.8$ & $<0.001$ \\
\hline Siegel's recovery (grade 1/2/3/4) & $65 / 29 / 15 / 44$ & $3 / 1 / 9 / 13$ & $<0.001$ \\
\hline
\end{tabular}

Data are presented as mean \pm standard deviation or $n(\%)$. FLAIR, fluid-attenuated inversion recovery; PTA, pure tone average 
Table 2. Data of matched patients

\begin{tabular}{lccc}
\hline & FLAIR-negative $(\mathrm{n}=23)$ & FLAIR-positive $(\mathrm{n}=23)$ & $p$-value \\
\hline Sex (male:female) & $10: 13$ & $17: 6$ & 0.071 \\
Age (year) & $52.0 \pm 13.4$ & $49.2 \pm 10.6$ & 0.438 \\
Treatment interval (day) & $3.5 \pm 3.1$ & $3.9 \pm 3.0$ & 0.666 \\
Intratympanic injection & $2(8.7)$ & $3(13.0)$ & $>0.999$ \\
MRI interval (day) & $7.0 \pm 6.1$ & $10.1 \pm 7.5$ & 0.133 \\
Initial PTA (dB) & $101.7 \pm 18.0$ & $104.3 \pm 17.0$ & 0.617 \\
Final PTA (dB) & $67.1 \pm 33.7$ & $76.0 \pm 25.3$ & 0.315 \\
Siegel's recovery (grade 1/2/3/4) & $3 / 4 / 6 / 10$ & $1 / 1 / 8 / 13$ & 0.324 \\
\hline
\end{tabular}

Data are presented as mean \pm standard deviation or $n$ (\%). FLAIR, fluid-attenuated inversion recovery; PTA, pure tone average

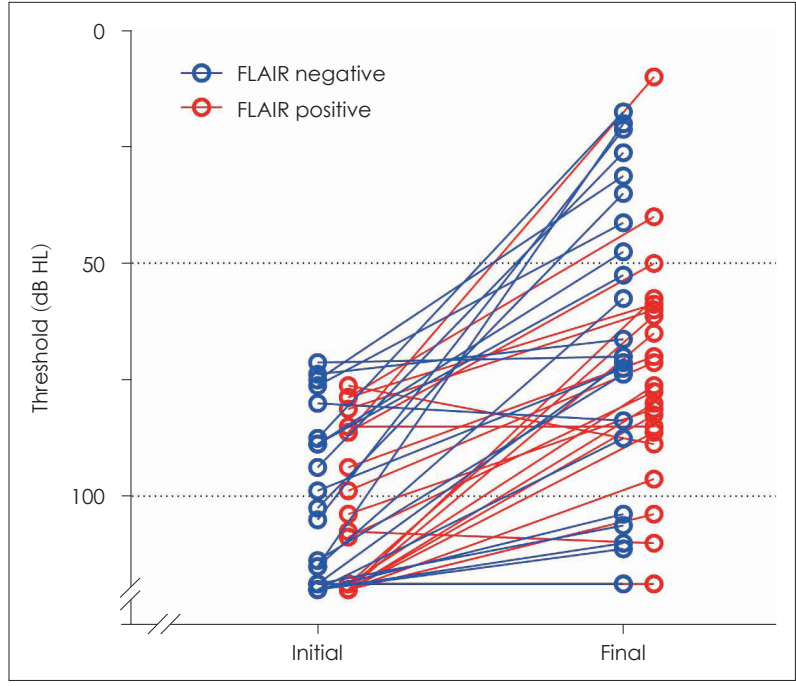

Fig. 1. Similar hearing recovery between matched groups. The average pure tone thresholds of individual patients were plotted on the graph. Empty circles connected to a line indicate the initial and final hearing thresholds of the same patient. FLAIR, fluid-attenuated inversion recovery.

pure tone threshold $(p=0.315)$ or the Siegel's recovery criteria $(p=0.324)$. Thirty percent of FLAIR-negative group and $9 \%$ of FLAIR-positive group showed improvement in grades 1 and 2 by Siegel's criteria, with no difference between the groups (odds ratio $=0.218,95 \%$ confidence interval $0.040-$ $1.192, p=0.135)$.

\section{Logistic regression analysis in patients with profound initial hearing}

Next, we tried to confirm the effect of each prognostic factor using logistic regression analysis. Because initial hearing can affect FLAIR MRI results, the analysis was conducted in both groups of the whole cohort $(\mathrm{n}=179)$ and patients with profound initial hearing $(n=56)$. As a dependent variable, hearing recovery was defined when Siegel's recovery level was 1 or 2. Multivariate logistic regression performed including age, initial hearing, FLAIR signal, treatment interval, and treatment

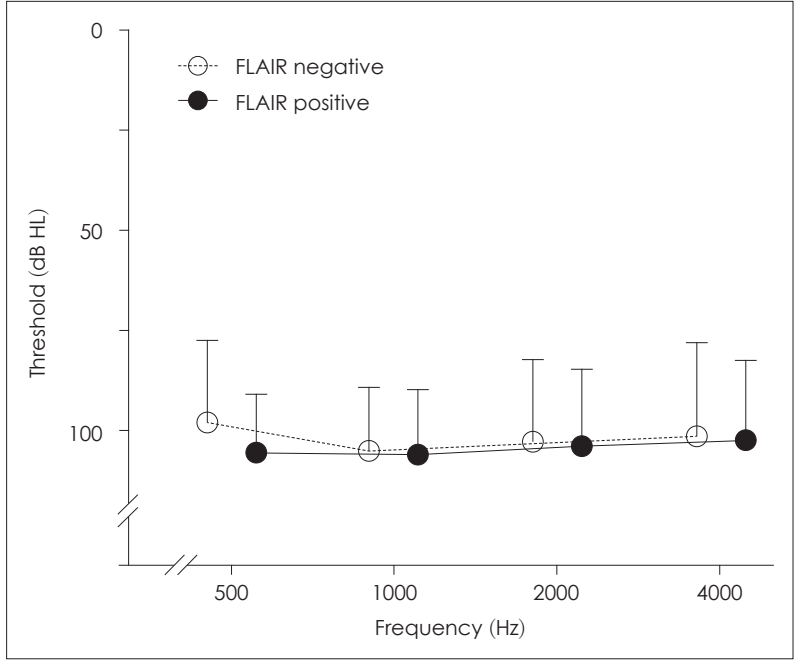

Fig. 2. Similar initial hearing thresholds at all frequencies in the matched groups. There was no difference between the groups at frequencies of $500,1000,2000$, and $4000 \mathrm{~Hz}$. The empty and black circles indicate the mean pure tone threshold of FLAIR-negative and FLAIR-positive groups, respectively. The error bar indicates standard deviation. FLAIR, fluid-attenuated inversion recovery.

type as independent variables (Table 3$)$. In the pre-matched 179 patients, FLAIR signal $(p=0.023)$ and initial hearing $(p<$ 0.001 ) were significantly associated with hearing recovery. In the profound hearing loss patients, only initial hearing was significantly associated with hearing recovery $(p=0.008)$.

\section{Discussion}

In our cohort of 179 patients, the cochlea increased signal in pre-contrast 3D FLAIR images positively correlated with the severity of SSNHL. The prognosis categorized by Siegel's criteria was also better in FLAIR-negative group. However, based on the matched analysis, the prognosis does not seem to be affected mainly by the signal difference in pre-contrast 3D FLAIR images. This result is supported by the logistic regression analysis of the profound hearing loss patients group. The positive relationship between initial hearing and FLAIR 
Table 3. Logistic regression analysis

\begin{tabular}{|c|c|c|c|c|c|}
\hline \multirow{2}{*}{ Independent variables } & \multirow{2}{*}{ Value } & \multicolumn{2}{|c|}{ Whole cohort $(n=179)$} & \multicolumn{2}{|c|}{ Profound hearing loss $(n=56)$} \\
\hline & & $B$ & $p$-value & B & p-value \\
\hline FLAIR signal & $1=$ increase, $2=$ symmetric & -1.393 & $0.023^{*}$ & 0.111 & 0.855 \\
\hline Age & Continuous & -0.009 & 0.499 & -0.072 & 0.100 \\
\hline Initial hearing & Continuous & -0.033 & $<0.001^{*}$ & -0.190 & $0.008^{*}$ \\
\hline Treatment interval & Continuous & -0.079 & 0.246 & -0.057 & 0.786 \\
\hline Steroid administration & $0=P O, 1=|T D|$ & -0.083 & 0.905 & 0.872 & 0.569 \\
\hline
\end{tabular}

Nagelkerke R2: whole cohort $=0.305$, profound $=0.488$. Classification accuracy: whole cohort $=74.7 \%$, profound $=87.3 \%$. ${ }^{*} p<0.05$. FLAIR, fluid-attenuated inversion recovery; PO, peroral administration; ITDI, intratympanic injection

signal increase likely causes overestimation of the prognostic value of pre-contrast 3D FLAIR image. In a clinical aspect, the prognostic value of pre-contrast 3D FLAIR image is weakened by this study. However, the importance of MRI in SSNHL patients should not be underestimated, as it is still an irreplaceable method for diagnosing vestibular schwannoma.

The results of this study agree with those of some previous studies, ${ }^{8,10)}$ but contrasts with others showing that 3D FLAIR images could predict the hearing recovery result of SSNHL patients. ${ }^{15-17)}$ The previously reported association of cochlear increased signal on 3D FLAIR images, and poor initial hearing was reproduced in the present study. Given that a high signal on 3D FLAIR images reflects methemoglobin and proteinaceous exudate, severely disrupted BLB might result in the severe SSNHL phenotype observed. Although the mechanism of SSNHL is not fully understood, it is very plausible that an inflammatory response is involved in its pathophysiology. Hair cell loss was shown to induce an inflammatory reaction in the cochlea in an animal model. ${ }^{18)}$ Another evidence could be the clinical advantage of corticosteroid therapy that reduces tissue damage associated with inflammation. ${ }^{19)}$ Severe inflammation can damage the BLB and introduce methemoglobin and proteinaceous exudate into the inner ear space. Nevertheless, disruption of the BLB seems to have a limited effect on cochlear function recovery, based on the result of this study. Since initial hearing was an important factor in predicting hearing recovery in other studies, hearing recovery might be affected by the severity of inflammation as well the severity of disruption to the BLB. However, how much exudate present in the inner ear space may not affect the hearing recovery in SSNHL patients, based on the results of the present study.

To the best of our knowledge, no study has yet investigated the prognostic value of cochlear abnormality on 3D FLAIR images between initial hearing-matched SSNHL groups. However, regression analysis was attempted in several studies to minimize confounding factors. Using a multivariate regression model, Yoshida, et al. ${ }^{15)}$ reported that the period to the initial visit, high cochlear signal, and initial audiogram were significantly correlated with the hearing outcome. Among the significant variables, the initial audiogram showed the most significant relationship. Lee, et al. ${ }^{10)}$ found, using a multivariate regression model, that the presence of a high-intensity signal on pre-contrast FLAIR images was not associated with the final hearing outcome. However, the initial hearing was the most significant factor affecting the final hearing outcome in their study. The results of this study also demonstrated that initial hearing is the most considerable variable to determine hearing recovery. In the logistic regression analysis before matching, FLAIR signal was also shown to affect the prognosis, as in other studies. After matching, however, only the initial hearing was significant. Considering that all of the included variables were hypothesized as independent in the regression models, the strong association between initial hearing and increased cochlear signal on 3D FLAIR images could lead to some bias in the results and overestimate the significance of 3D FLAIR image findings.

The cochlear abnormality rate on 3D FLAIR images was very low (14.5\%), and the initial hearing in FLAIR-positive group was very poor $(100.1 \pm 22.6 \mathrm{~dB})$. This may have resulted from the differences in how patients were selected for MRI as well as the differences in inclusion criteria, since most studies, including the current one, were conducted retrospectively. Moreover, the MRI settings, treatment modality, and the definition of signal increase may have differed between studies. The poor initial hearing level in the present study might be an important limitation, as poor initial hearing in itself is a poor prognostic factor for hearing recovery. To control for this factor, we matched the patients by initial hearing and treatment interval.

Meanwhile, post-contrast 3D FLAIR MRI findings showed high sensitivity and encouraging prognostic value in several 
studies. $^{20,21)}$ An enhancement in the post-contrast 3D FLAIR MRI can demonstrate loosening of the BLB. Although both pre-contrast 3D FLAIR MRI and post-contrast 3D FLAIR MRI can reflect on the integrity of BLB, care should be taken to differentiate between them since the visualized pathology is different. Based on the results of the present study, exuded materials in the inner ear space have a limited effect on hearing recovery. In contrast, post-contrast 3D FLAIR MRI can demonstrate the degree of BLB damage more directly by showing the leakage of the contrast medium. Therefore, enhancement in post-contrast 3D FLAIR MRI might be more strongly associated with the initial hearing and hearing recovery. However, the prognostic value of post-contrast 3D FLAIR MRI should also be carefully interpreted since a strong correlation with initial hearing could be a confounding factor, as shown in the present study.

The present study had some limitations. First, the interpretation of MRI images was subjectively assessed, and an objective interpretation using the software would guarantee better reproducibility and more numbers of matched pairs. Moreover, the subjective assessment of MRI images might have led to underestimation of the asymmetry, which may be associated with the low rate of cochlea asymmetry in this study. Another limitation was the selectively conducted MRI. Although all of the SSNHL patients were routinely recommended for MRI, it was performed in 546 of 4643 patients mainly due to financial reasons. The public health insurance system in South Korea covers the cost for MRI only if the patient has an average pure-tone threshold worse than $40 \mathrm{~dB}$. This may bias the study population to more severe patients and can affect the result. The reduced number after matching can also cause an underestimation of the results. Thus, a largescale study should be conducted in the future. Nevertheless, it seems obvious that initial hearing is a more significant predictor of hearing recovery compared to FLAIR MRI.

In conclusion, the abnormal cochlear signal on 3D FLAIR MRI was associated with poor initial hearing. However, after matching for initial hearing, it had no association with the hearing recovery outcome. The exuded material in the inner ear space seems to have a limited influence on SSNHL prognosis, but it might reflect on the severity of the disease.

\section{Acknowledgments}

This study was supported by a faculty research grant from the Yonsei University College of Medicine (6-2020-0153 granted to SHB) and the Basic Science Research Program through the National Research Foundation of Korea (NRF) funded by the Ministry of
Education (NRF-2020R1I1A1A01067241 granted to SHB).

\section{Author Contribution}

Conceptualization: Ara Cho, Seong Hoon Bae. Data curation: Geun Cheol Shin, Min Cheol Jeong, Beom Seok Sohn, Seong Hoon Bae. Formal analysis: Geun Cheol Shin, Seong Hoon Bae. Funding acquisition: Seong Hoon Bae. Project administration: Seong Hoon Bae. Resources: Ara Cho, Seong Hoon Bae. Supervision: Seong Hoon Bae. Visualization: Beom Seok Sohn, Seong Hoon Bae. Writing - original draft: Geun Cheol Shin. Writing_review \& editing: Seong Hoon Bae.

\section{ORCIDs}

Seong Hoon Bae https://orcid.org/0000-0001-9243-9392

Geun Cheol Shin https://orcid.org/0000-0003-0341-4711

Ara Cho

https://orcid.org/0000-0002-1863-4883

\section{REFERENCES}

1) Rauch SD. Clinical practice. Idiopathic sudden sensorineural hearing loss. N Engl J Med 2008;359(8):833-40.

2) Schreiber BE, Agrup C, Haskard DO, Luxon LM. Sudden sensorineural hearing loss. Lancet 2010;375(9721):1203-11.

3) Fitzgerald DC, McGuire JF. Intratympanic steroids for idiopathic sudden sensorineural hearing loss. Ann Otol Rhinol Laryngol 2007;116(4):253-6.

4) Wei BP, Stathopoulos D, O’Leary S. Steroids for idiopathic sudden sensorineural hearing loss. Cochrane Database Syst Rev 2013; 2013(7):CD003998.

5) Banerjee A, Parnes LS. Intratympanic corticosteroids for sudden idiopathic sensorineural hearing loss. Otol Neurotol 2005;26(5): 878-81.

6) Jo SY, Lee S, Eom TH, Jeun ES, Cho HH, Cho YB. Outcomes of severe to profound idiopathic sudden sensorineural hearing loss. Clin Exp Otorhinolaryngol 2015;8:206-10.

7) Conte G, Di Berardino F, Sina C, Zanetti D, Scola E, Gavagna C, et al. MR imaging in sudden sensorineural hearing loss. Time to talk. AJNR Am J Neuroradiol 2017;38(8):1475-9.

8) Berrettini S, Seccia V, Fortunato S, Forli F, Bruschini L, Piaggi P, et al. Analysis of the 3-dimensional fluid-attenuated inversionrecovery (3D-FLAIR) sequence in idiopathic sudden sensorineural hearing loss. JAMA Otolaryngol Head Neck Surg 2013;139(5):45664.

9) Ryu IS, Yoon TH, Ahn JH, Kang WS, Choi BS, Lee JH, et al. Three-dimensional fluid-attenuated inversion recovery magnetic resonance imaging in sudden sensorineural hearing loss: correlations with audiologic and vestibular testing. Otol Neurotol 2011;32(8): 1205-9.

10) Lee HY, Jung SY, Park MS, Yeo SG, Lee SY, Lee SK. Feasibility of three-dimensional fluid-attenuated inversion recovery magnetic resonance imaging as a prognostic factor in patients with sudden hearing loss. Eur Arch Otorhinolaryngol 2012;269(8):1885-91.

11) Byl FM Jr. Sudden hearing loss: Eight years' experience and suggested prognostic table. Laryngoscope 1984;94(5 Pt 1):647-61.

12) Xenellis J, Karapatsas I, Papadimitriou N, Nikolopoulos T, Maragoudakis P, Tzagkaroulakis M, et al. Idiopathic sudden sensorineural hearing loss: Prognostic factors. J Laryngol Otol 2006;120(9):718-24.

13) Mattox DE, Simmons FB. Natural history of sudden sensorineural hearing loss. Ann Otol Rhinol Laryngol 1977;86(4 Pt 1):463-80.

14) Siegel LG. The treatment of idiopathic sudden sensorineural hearing loss. Otolaryngol Clin North Am 1975;8(2):467-73.

15) Yoshida $T$, Sugiura $M$, Naganawa $S$, Teranishi $M$, Nakata $S$, 
Nakashima T. Three-dimensional fluid-attenuated inversion recovery magnetic resonance imaging findings and prognosis in sudden sensorineural hearing loss. Laryngoscope 2008;118(8): 1433-7.

16) Zhu H, Ou Y, Fu J, Zhang Y, Xiong H, Xu Y. A comparison of inner ear imaging features at different time points of sudden sensorineural hearing loss with three-dimensional fluid-attenuated inversion recovery magnetic resonance imaging. Eur Arch Otorhinolaryngol 2015;272(10):2659-65.

17) Lee JI, Yoon RG, Lee JH, Park JW, Yoo MH, Ahn JH, et al. Prognostic value of labyrinthine 3D-FLAIR abnormalities in idiopathic sudden sensorineural hearing loss. AJNR Am J
Neuroradiol 2016;37(12):2317-22.

18) Wood MB, Zuo J. The contribution of immune infiltrates to ototoxicity and cochlear hair cell loss. Front Cell Neurosci 2017; 11:106.

19) Trune DR, Canlon B. Corticosteroid therapy for hearing and balance disorders. Anat Rec (Hoboken) 2012;295(11):1928-43.

20) Byun H, Chung JH, Lee SH, Park CW, Park DW, Kim TY. The clinical value of 4-hour delayed-enhanced 3D-FLAIR MR images in sudden hearing loss. Clin Otolaryngol 2019;44(3):336-42.

21) Wang J, Ren T, Sun W, Liang Q, Wang W. Post-contrast 3D-FLAIR in idiopathic sudden sensorineural hearing loss. Eur Arch Otorhinolaryngol 2019;276:1291-9. 